\title{
Progress Report on Computing Excited-State Hadron Masses in Lattice QCD
}

\author{
C. Morningstar*, A. Bell, J. Bulava ${ }^{\dagger}$, J. Foley**, K.J. Juge ${ }^{\ddagger}$, D. Lenkner* \\ and C.H. Wong* \\ * Dept. of Physics, Carnegie Mellon University, Pittsburgh, PA 15213, USA \\ ${ }^{\dagger}$ NIC, DESY, Platanenallee 6, D-15738, Zeuthen, Germany \\ ${ }^{* *}$ Dept. of Physics and Astronomy, University of Utah, Salt Lake City, UT 84112, USA \\ ${ }^{\ddagger}$ Dept. of Physics, University of the Pacific, Stockton, CA 95211, USA
}

\begin{abstract}
Our progress in computing the spectrum of excited baryons and mesons in lattice QCD is described. Sets of spatially-extended hadron operators with a variety of different momenta are used. A new method of stochastically estimating the low-lying effects of quark propagation is utilized which allows reliable determinations of temporal correlations of both single-hadron and multi-hadron operators. The method is tested on the isoscalar mesons in the scalar, pseudoscalar, and vector channels, and on the two-pion system of total isospin $I=0,1,2$.
\end{abstract}

Keywords: Lattice QCD, hadron spectroscopy

PACS: $12.38 . \mathrm{Gc}, 11.15 . \mathrm{Ha}, 12.39 . \mathrm{Mk}$

We are currently carrying out computations of the excitation spectrum of QCD in finite volume with ab initio Markov-chain Monte Carlo path integrations on anisotropic space-time lattices. Our first results using two flavors of dynamical quarks were reported in Ref. [1], and our most recent results can be found in Ref. [2]. Such calculations are very challenging. Computational limitations cause simulations to be done with quark masses that are unphysically large, leading to pion masses that are especially heavier than observed. The use of carefully designed quantum field operators is crucial for accurate determinations of low-lying energies. To study a particular state of interest, the energies of all states lying below that state must first be extracted, and as the pion gets lighter in lattice QCD simulations, more and more multi-hadron states lie below the masses of the excited resonances. The evaluation of correlations involving multi-hadron operators contains new challenges since not only must initial to final time quark propagation be included, but also final to final time quark propagation must be incorporated.

The use of operators whose correlation functions $C(t)$ attain their asymptotic form as quickly as possible is crucial for reliably extracting excited hadron masses. An important ingredient in constructing such hadron operators is the use of smeared fields. Operators constructed from smeared fields have dramatically reduced mixings with the high frequency modes of the theory. Both link-smearing[3] and quark-field smearing[4] must be applied. Since excited hadrons are expected to be large objects, the use of spatially extended operators is another key ingredient in the operator design and implementation. A more detailed discussion of these issues can be found in Ref. [5].

A large effort was undertaken during the last two years to select optimal sets of baryon and meson operators in a large variety of isospin sectors and for zero momentum and 

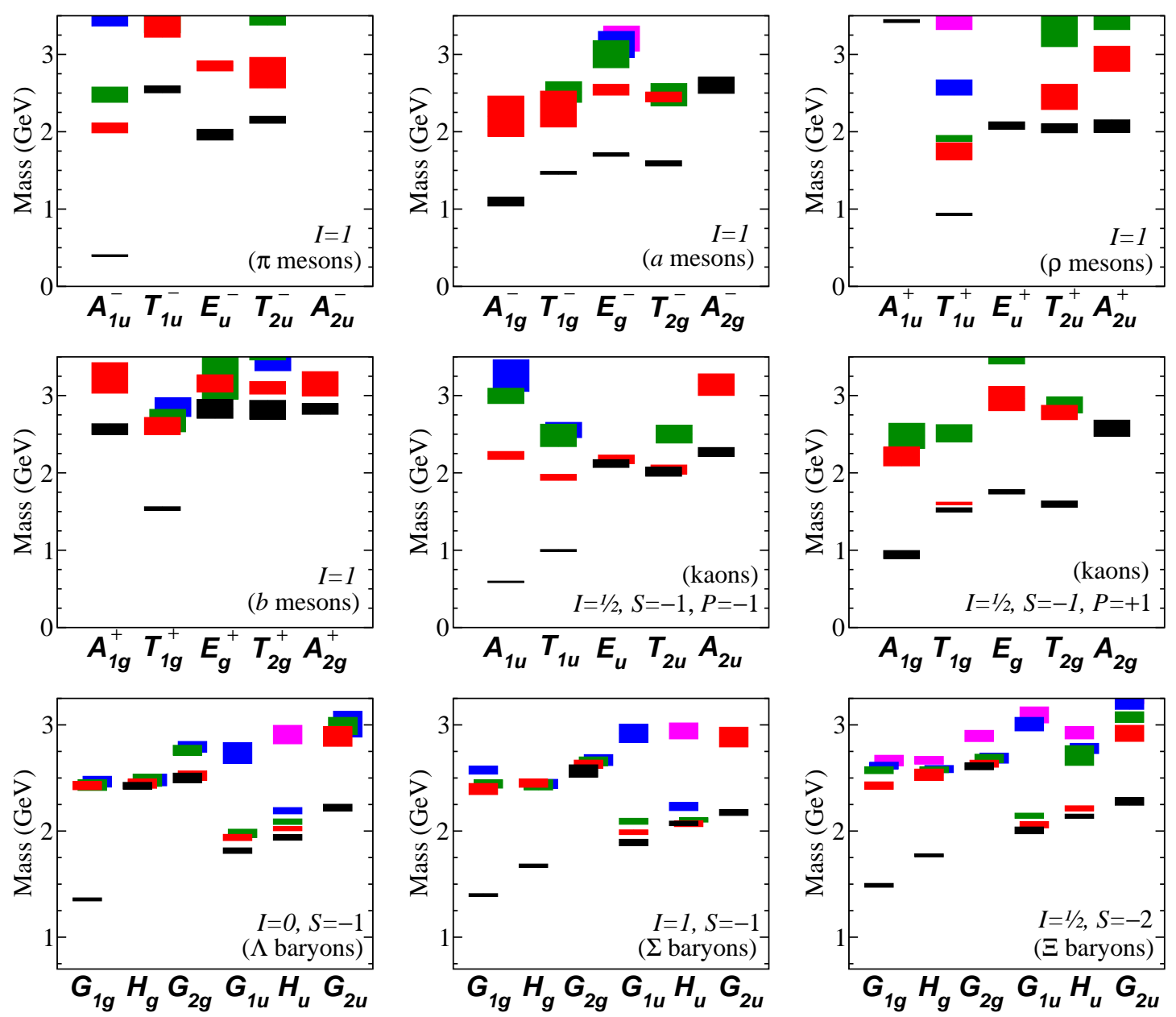

FIGURE 1. Hadron operator selection: low-statistics simulations have been performed to study the hundreds of single-hadron operators produced by our group-theoretical construction. A "pruning" procedure was followed in each channel to select good sets of between six to a dozen operators. The plots above show the stationary-state energies extracted to date from correlation matrices of the finally selected single-hadron operators. Results were obtained using between 50 to 100 configurations on a $16^{3} \times 128$ anisotropic lattice for $N_{f}=2+1$ quark flavors with spacing $a_{s} \sim 0.12 \mathrm{fm}, a_{s} / a_{t} \sim 3.5$, and quark masses such that $m_{\pi} \sim 400 \mathrm{MeV}$. Each box indicates the energy of one stationary state; the vertical height of each box indicates the statistical error.

non-zero on-axis, planar-diagonal, and cubic-diagonal momenta. Low-statistics Monte Carlo computations were done to accomplish these operator selections using between 50 to 100 configurations on a $16^{3} \times 128$ anisotropic lattice for $N_{f}=2+1$ quark flavors with spacing $a_{s} \sim 0.12 \mathrm{fm}, a_{s} / a_{t} \sim 3.5$, and quark masses such that the pion has mass around $400 \mathrm{MeV}$. Stationary-state energies using the finally selected operator sets are shown in Fig. 1. The nucleon, $\Delta, \Xi, \Sigma$, and $\Lambda$ baryons were studied, and light isovector and kaon mesons were investigated. Hundreds of operators were studied, and optimal sets containing eight or so operators in each symmetry channel were found. Future computations will focus solely on the operators in the optimal sets.

A comprehensive picture of resonances requires that we go beyond a knowledge of 

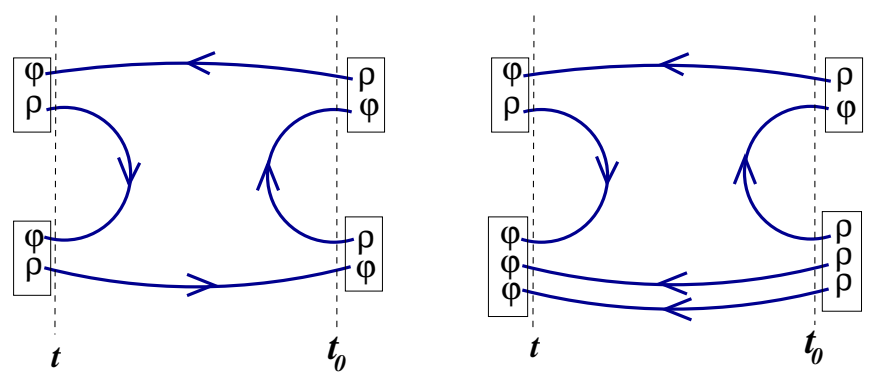

FIGURE 2. Diagrams of multi-hadron correlators that require having $\rho$ noise sources on the later time $t$. Solution vectors are denoted by $\varphi$. (Left) A two-meson correlator. (Right) The correlator of a baryonmeson system.

the ground state mass in each symmetry channel and obtain the masses of the lowest few states in each channel. This necessitates the use of matrices of correlation functions[6, 7]. Rather than evaluating a single correlator $C(t)$, we determine a matrix of correlators $C_{i j}(t)=\left\langle O_{i}(t) O_{j}^{\dagger}\left(t_{0}\right)\right\rangle$, where $\left\{O_{i} ; i=1, \ldots, N\right\}$ are a basis of interpolating operators with given quantum numbers. We then solve the generalized eigenvalue equation $C(t) u=\lambda\left(t, t_{0}\right) C\left(t_{0}\right) u$ to obtain a set of real (ordered) eigenvalues $\lambda_{n}\left(t, t_{0}\right)$, where $\lambda_{0} \geq \lambda_{1} \geq \ldots \geq \lambda_{N-1}$. At large Euclidean times, these eigenvalues then delineate between the different masses $\lambda_{n}\left(t, t_{0}\right) \longrightarrow e^{-M_{n}\left(t-t_{0}\right)}+O\left(e^{-\Delta M_{n}\left(t-t_{0}\right)}\right)$, where $\Delta M_{n}=\min \left\{\left|M_{n}-M_{i}\right|: i \neq n\right\}$. The eigenvectors $u$ are orthogonal with metric $C\left(t_{0}\right)$, and the eigenvectors yield information about the structure of the states.

To study a particular eigenstate of interest with this method, all eigenstates lying below that state must first be extracted, and as the pion gets lighter in lattice QCD simulations, more and more multi-hadron states will lie below the excited resonances. A good baryon-meson operator of total zero momentum is typically a superposition of local interpolating fields at all sites on a time slice of the lattice. In the evaluation of the temporal correlations of such a multi-hadron operator, it is not possible to completely remove all summations over the spatial sites on the source time-slice using translation invariance. Hence, the need for estimates of the quark propagators from all spatial sites on a time slice to all spatial sites on another time slice cannot be sidestepped. Some correlators will involve diagrams with quark lines originating at the sink time $t$ and terminating at the same sink time $t$ (see Fig. 2), so quark propagators involving a large number of starting times $t$ must also be handled.

Finding better ways to stochastically estimate slice-to-slice quark propagators is crucial to the success of our excited-state hadron spectrum project at lighter pion masses. We have developed and tested a new scheme which combines a new way of smearing the quark field with a new way of introducing noise. The new quark-field smearing scheme, called Laplacian Heaviside (LapH), has been described in Ref. [4] and is defined by

$$
\widetilde{\psi}(x)=\Theta\left(\sigma_{s}^{2}+\widetilde{\Delta}\right) \psi(x),
$$

where $\widetilde{\Delta}$ is the three-dimensional covariant Laplacian in terms of the stout-smeared gauge field and $\sigma_{s}$ is the smearing parameter. The gauge-covariant Laplacian operator is ideal for smearing the quark field since it is one of the simplest operators that locally 
averages the field in such a way that all relevant symmetry transformation properties of the original field are preserved. Let $V_{\Delta}$ denote the unitary matrix whose columns are the eigenvectors of $\widetilde{\Delta}$, and let $\Lambda_{\Delta}$ denote a diagonal matrix whose elements are the eigenvalues of $\widetilde{\Delta}$ such that $\widetilde{\Delta}=V_{\Delta} \Lambda_{\Delta} V_{\Delta}^{\dagger}$. The LapH smearing matrix is then given by $S=V_{\Delta} \Theta\left(\sigma_{s}^{2}+\Lambda_{\Delta}\right) V_{\Delta}^{\dagger}$. Let $V_{s}$ denote the matrix whose columns are in one-to-one correspondence with the eigenvectors associated with the $N_{v}$ lowest-lying eigenvalues of $-\widetilde{\Delta}$ on each time slice. Then our LapH smearing matrix is well approximated by the Hermitian matrix $S=V_{s} V_{s}^{\dagger}$. Evaluating the temporal correlations of our hadron operators requires combining Dirac matrix elements associated with various quark lines $Q$. Since we construct our hadron operators out of covariantly-displaced, smeared quark fields, each and every quark line involves the following product of matrices:

$$
Q=D^{(j)} S M^{-1} S D^{(k) \dagger},
$$

where $D^{(i)}$ is a gauge-covariant displacement of type $i$. An exact treatment of such a quark line is very costly, so we resort to stochastic estimation.

Random noise vectors $\eta$ whose expectations satisfy $E\left(\eta_{i}\right)=0$ and $E\left(\eta_{i} \eta_{j}^{*}\right)=\delta_{i j}$ are useful for stochastically estimating the inverse of a large matrix $M$ as follows [8]. Assume that for each of $N_{R}$ noise vectors, we can solve the following linear system of equations: $M X^{(r)}=\eta^{(r)}$ for $X^{(r)}$. Then $X^{(r)}=M^{-1} \eta^{(r)}$, and $E\left(X_{i} \eta_{j}^{*}\right)=M_{i j}^{-1}$ so that a Monte Carlo estimate of $M_{i j}^{-1}$ is given by $M_{i j}^{-1} \approx \lim _{N_{R} \rightarrow \infty} \frac{1}{N_{R}} \sum_{r=1}^{N_{R}} X_{i}^{(r)} \eta_{j}^{(r) *}$. Unfortunately, this equation usually produces stochastic estimates with variances which are much too large to be useful. Variance reduction is done by diluting the noise vectors. A given dilution scheme can be viewed as the application of a complete set of projection operators $P^{(a)}$. Define $\eta_{k}^{[a]}=P_{k k^{\prime}}^{(a)} \eta_{k^{\prime}}$, and further define $X^{[a]}$ as the solution of $M_{i k} X_{k}^{[a]}=\eta_{i}^{[a]}$, then we have

$$
M_{i j}^{-1} \approx \lim _{N_{R} \rightarrow \infty} \frac{1}{N_{R}} \sum_{r=1}^{N_{R}} \sum_{a} X_{i}^{(r)[a]} \eta_{j}^{(r)[a] *} .
$$

The use of $Z_{4}$ noise ensures zero variance in the diagonal elements $E\left(\eta_{i} \eta_{i}^{*}\right)$.

The effectiveness of the variance reduction depends on the projectors chosen. With LapH smearing, noise vectors $\rho$ can be introduced only in the LapH subspace. The noise vectors $\rho$ now have spin, time, and Laplacian eigenmode number as their indices. Color and space indices get replaced by Laplacian eigenmode number. Again, each component of $\rho$ is a random $Z_{4}$ variable so that $E(\rho)=0$ and $E\left(\rho \rho^{\dagger}\right)=I_{d}$. Dilution projectors $P^{(b)}$ are now matrices in the LapH subspace. In the stochastic LapH method, a quark line on a gauge configuration is evaluated as follows:

$$
\begin{aligned}
Q & =D^{(j)} S M^{-1} S D^{(k) \dagger}=D^{(j)} S M^{-1} V_{s} V_{s}^{\dagger} D^{(k) \dagger} \\
& =\sum_{b} D^{(j)} S M^{-1} V_{s} P^{(b)} P^{(b) \dagger} V_{s}^{\dagger} D^{(k) \dagger} \\
& =\sum_{b} D^{(j)} S M^{-1} V_{s} P^{(b)} E\left(\rho \rho^{\dagger}\right) P^{(b) \dagger} V_{s}^{\dagger} D^{(k) \dagger} \\
& =\sum_{b} E\left(D^{(j)} S M^{-1} V_{s} P^{(b)} \rho\left(D^{(k)} V_{s} P^{(b)} \rho\right)^{\dagger}\right) .
\end{aligned}
$$


For a noise vector labelled by index $r$, displaced-smeared-diluted quark source and quark sink vectors can be defined by

$$
\begin{aligned}
& \rho^{(r)[b](j)}=D^{(j)} V_{S} P^{(b)} \rho^{(r)}, \\
& \varphi^{(r)[b](j)}=D^{(j)} S M^{-1} V_{S} P^{(b)} \rho^{(r)},
\end{aligned}
$$

and each quark line on a given gauge configuration can be estimated using

$$
Q_{u v} \approx \frac{1}{N_{R}} \sum_{r=1}^{N_{R}} \sum_{b} \varphi_{u}^{(r)[b](j)} \rho_{v}^{(r)[b](k) *},
$$

where the subscripts $u, v$ are compound indices combining space, time, color, and spin.

Our dilution projectors are products of time dilution, spin dilution, and Laph eigenvector dilution projectors. For each type (time, spin, Laph eigenvector) of dilution, we studied four different dilution schemes. Let $N$ denote the dimension of the space of the dilution type of interest. For time dilution, $N=N_{t}$ is the number of time slices on the lattice. For spin dilution, $N=4$ is the number of Dirac spin components. For Laph eigenvector dilution, $N=N_{v}$ is the number of eigenvectors retained. The four schemes we studied are defined below:

$$
\begin{aligned}
& P_{i j}^{(a)}=\delta_{i j}, \quad a=0, \quad \text { (no dilution) } \\
& P_{i j}^{(a)}=\delta_{i j} \delta_{a i}, \quad a=0, \ldots, N-1 \quad \text { (full dilution) } \\
& P_{i j}^{(a)}=\delta_{i j} \delta_{a,\lfloor K i / N\rfloor} a=0, \ldots, K-1, \quad(\text { block-K) } \\
& P_{i j}^{(a)}=\delta_{i j} \delta_{a, i \bmod K} a=0, \ldots, K-1, \quad(\text { interlace- } K \text { ) }
\end{aligned}
$$

where $i, j=0, \ldots, N-1$, and we assume $N / K$ is an integer. We use a triplet (T, S, L) to specify a given dilution scheme, where " $T$ " denote time, " $S$ " denotes spin, and " $L$ " denotes Laph eigenvector dilution. The schemes are denoted by 1 for no dilution, $\mathrm{F}$ for full dilution, and $\mathrm{B} K$ and $\mathrm{I} K$ for block- $K$ and interlace- $K$, respectively. For example, full time and spin dilution with interlace-8 Laph eigenvector dilution is denoted by (TF, SF, LI8). Introducing diluted noise in this way produces correlation functions with significantly reduced variances, yielding nearly an order of magnitude reduction in the statistical error over previous methods. The volume dependence of this new method was found to be very mild, allowing the method to be useful on large lattices. For all forwardtime quark lines, we use dilution scheme (TF, SF, LI8), and for all same-sink-time quark lines, we use (TI16, SF, LI8).

Results for three isoscalar mesons are shown in Fig. 3. Such mesons are notoriously difficult to study in lattice QCD, but the new method appears to produce estimates of their temporal correlations with unprecedented accuracy. We also tested our method by evaluating the energies of various two-pion states, in particular, the total isospin $I=0,1,2$ cases for various relative momenta. The results are shown in Fig. 4. These plots suggest that evaluating correlation functions involving our multi-hadron operators will be feasible with the stochastic LapH method.

We are currently carrying out these spectrum computations on $24^{3} \times 128$ and $32^{3} \times$ 256 anisotropic lattices with spatial spacing $a_{s} \sim 0.12 \mathrm{fm}$ and aspect ratio $a_{s} / a_{t} \sim 3.5$, 

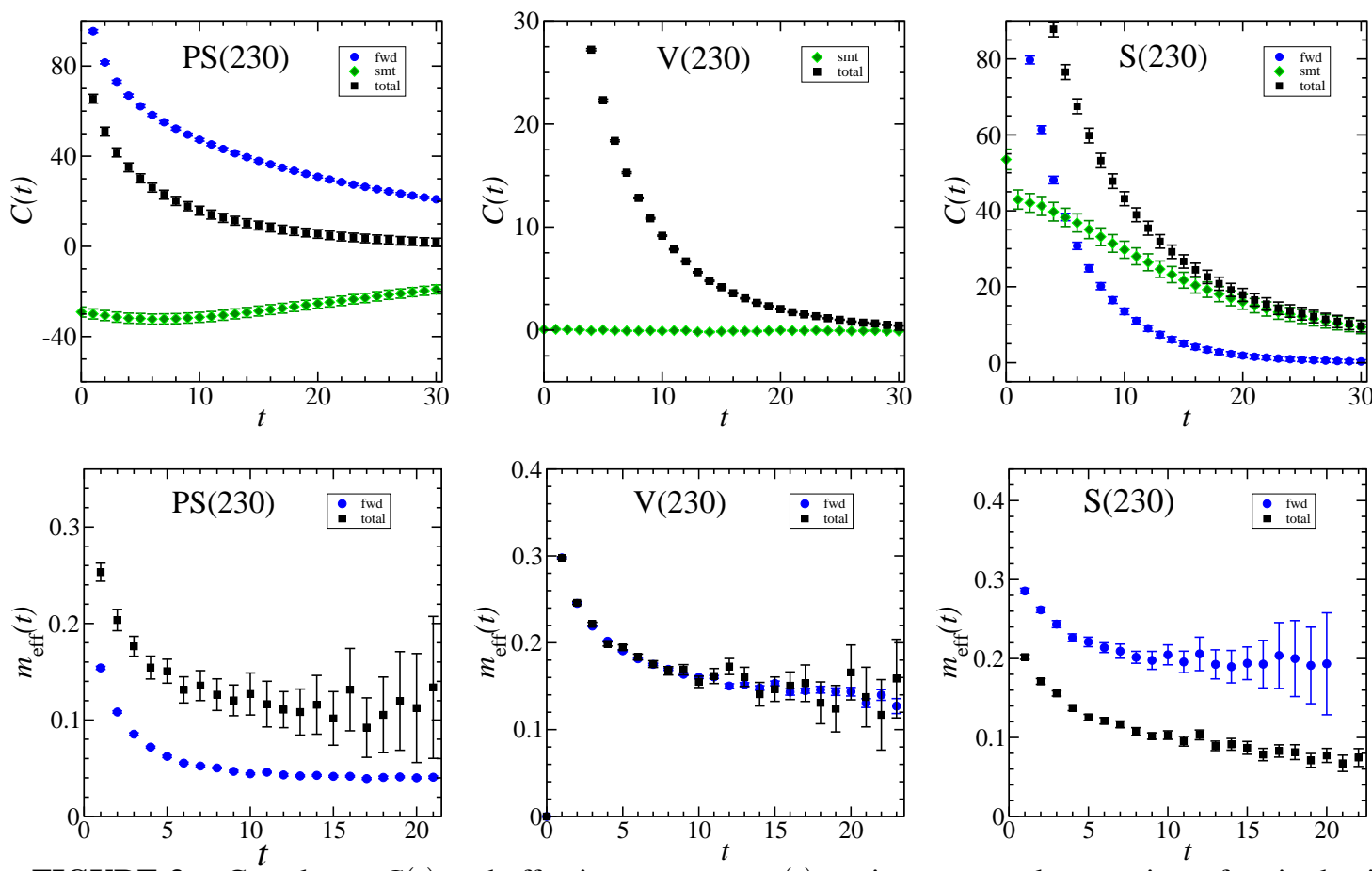

FIGURE 3. Correlators $C(t)$ and effective masses $m_{\mathrm{eff}}(t)$ against temporal separation $t$ for single-site operators in the the isoscalar pseudoscalar (PS), vector (V), and scalar (S) channels. Results were obtained using 198 configurations with $N_{f}=2+1$ flavors of quark loops on a $24^{3} \times 128$ anisotropic lattice with spacing $a_{s} \sim 0.12 \mathrm{fm}$ and aspect ratio $a_{s} / a_{t} \sim 3.5$ for a pion mass $m_{\pi} \sim 230 \mathrm{MeV}$. In the legends, "fwd" refers to contributions from the diagram containing only forward-time source-to-sink quark lines, "smt" refers to contributions from the diagram containing only quark lines that originate and terminate at the same time. For the $\sigma$ channel, the "smt" contribution has a vacuum expectation value subtraction. Forward-time quark lines use dilution scheme (TF, SF, LI8) and same-time quark lines use (TI16, SF, LI8).

where $a_{t}$ is the temporal spacing, for pion masses $m_{\pi} \sim 400 \mathrm{MeV}$ and $m_{\pi} \sim 230 \mathrm{MeV}$. The calculations proceed in several steps: (a) generation of gauge-field configurations using the Monte Carlo method; (b) computation of quark sinks for various noises and dilution projectors using the configurations from the first step; (c) computation of the meson and baryon sources and sinks using the quark sinks from the second step; (d) evaluation of the correlators using the hadron sinks; (e) analysis of the correlators to extract the energies. Our results for the QCD stationary-state energies should appear soon.

This work was supported by the U.S. National Science Foundation under awards PHY0510020, PHY-0653315, PHY-0704171, PHY-0969863, and PHY-0970137, and through TeraGrid resources provided by the Pittsburgh Supercomputer Center, the Texas Advanced Computing Center, and the National Institute for Computational Sciences under grant numbers TG-PHY100027 and TG-MCA075017. The USQCD QDP++/Chroma library[9] was used in developing the software for the calculations reported here. We thank our colleagues within the Hadron Spectrum Collaboration. 

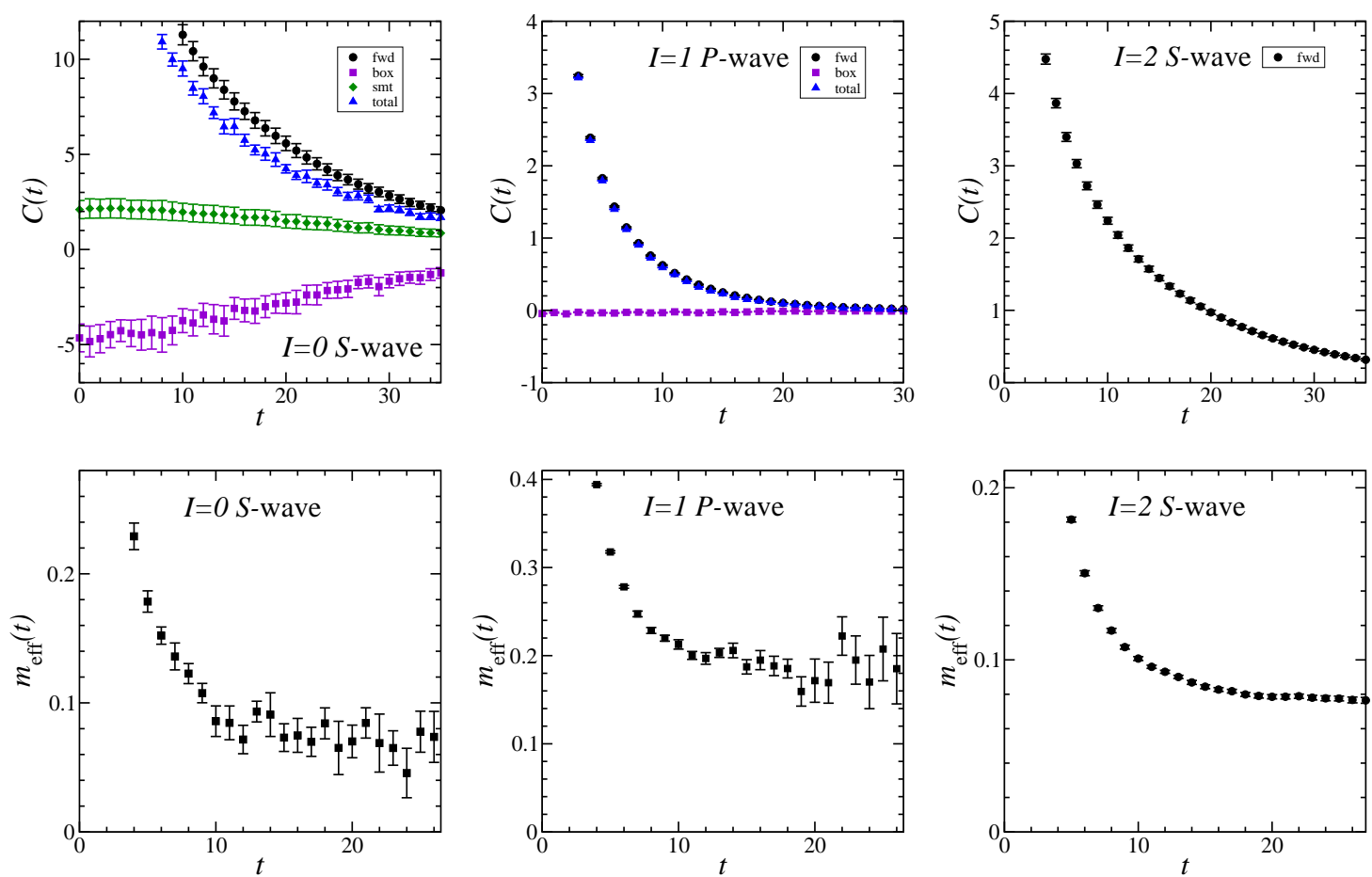

FIGURE 4. Correlators $C(t)$ and effective masses $m_{\mathrm{eff}}(t)$ against temporal separation $t$ for two-pion operators with total isospin $I=0,1,2$ and zero total momentum. $S$-wave results have zero relative momentum, $P$-wave has minimal non-zero on-axis relative momenta. Results were obtained using 584 configurations with $N_{f}=2+1$ flavors of quark loops on a $24^{3} \times 128$ anisotropic lattice with spacing $a_{s} \sim 0.12 \mathrm{fm}$ and aspect ratio $a_{s} / a_{t} \sim 3.5$ for a pion mass $m_{\pi} \sim 230 \mathrm{MeV}$. In the legends, "fwd" refers to contributions from diagrams containing only forward-time source-to-sink quark lines, "smt" refers to contributions from diagrams containing only quark lines that originate and terminate at the same time, and "box" refers to diagrams containing both kinds of quark lines. Forward-time quark lines use dilution scheme (TF, SF, LI8) and same-time quark lines use (TI16, SF, LI8).

\section{REFERENCES}

1. J. Bulava, R.G. Edwards, E. Engelson, J. Foley, B. Joo, A. Lichtl, H.-W. Lin, N. Mathur, C. Morningstar, D.G. Richards, S. Wallace, Phys. Rev. D 79, 034505 (2009).

2. J. Bulava, R.G. Edwards, E. Engelson, B. Joo, H-W. Lin, C. Morningstar, D.G. Richards, S.J. Wallace, Phys. Rev. D82, 014507 (2010).

3. C. Morningstar and M. Peardon, Phys. Rev. D 69, 054501 (2004).

4. M. Peardon, J. Bulava, J. Foley, C. Morningstar, J. Dudek, R. Edwards, B. Joo, H-W. Lin, D. Richards, K.J. Juge, Phys. Rev. D 80, 054506 (2009).

5. S. Basak, R.G. Edwards, G.T. Fleming, U.M. Heller, C. Morningstar, D. Richards, I. Sato, S. Wallace, Phys. Rev. D72, 094506 (2005).

6. C. Michael, Nucl. Phys. B 259, 58 (1985).

7. M. Lüscher and U. Wolff, Nucl. Phys. B339, 222 (1990).

8. J. Foley, K.J. Juge, A. O'Cais, M. Peardon, S. Ryan, J. Skullerud, Comput. Phys. Commun. 172, 145 (2005).

9. R.G. Edwards and B. Joo, Nucl. Phys. B (Proc. Suppl.) 140, 832 (2005). 